\title{
Proteomic Analysis of the Luteal Endometrial Secretome
}

\author{
Jessica G. Scotchie, MD, Marc A. Fritz, MD, Mihaela Mocanu, MS, Bruce A. Lessey, MD, \\ PhD, and Steven L. Young, MD, PhD \\ Division of Reproductive Endocrinology and Infertility, Department of Obstetrics \& Gynecology, \\ University of North Carolina, Chapel Hill, North Carolina, (JGS, MAF, SLY); UNC-Duke Michael \\ Hooker Proteomics Center, University of North Carolina, Chapel Hill, North Carolina, (MM); and \\ Reproductive Endocrinology and Infertility, Department of Obstetrics \& Gynecology, Greenville \\ Hospital System, Greenville, South Carolina, (BAL)
}

\section{Abstract}

Endometrium attains a secretory architecture in preparation for embryo implantation, but the identity of most endometrial secretory products remains unknown. Our objective was to characterize the endometrial secretome and compare protein expression between prereceptive (luteinizing hormone $[\mathrm{LH}]+4)$ receptive $(\mathrm{LH}+9)$ and phase endometrium. Endometrial lavage was performed in 11 participants and analyzed by difference gel electrophoresis (DIGE). LH+4 and LH+9 specimens were labeled with cyanine fluorescent dyes $\mathrm{Cy} 3$ and $\mathrm{Cy} 5$ tags, respectively, and combined. Proteins were separated using 2-dimensional gel electrophoresis, isolated, trypsin-digested, and subjected to mass spectrometry. In all, 152 proteins were identified; 82 were differentially expressed. Most proteins with increased expression on $\mathrm{LH}+9$ functioned in host defense, while proteins with decreased expression had many functions. A total of 14 proteins had changes suggesting altered posttranslational modification. This article describes the first application of proteomic analysis to endometrial secretions, allowing identification of novel endometrial proteins as well as those differentially secreted in prereceptive and receptive phases.

\section{Keywords}

Proteomics; endometrium; luteal phase; secretome; endometrial receptivity; fibrinogen gamma

\section{INTRODUCTION}

\begin{abstract}
Embryo implantation is necessary for survival of the human embryo and abnormalities in the implantation process are thought to cause infertility, pregnancy loss, and reduced success of in vitro fertilization (IVF) therapy. ${ }^{1,2}$ The endometrium becomes receptive to embryo implantation only during the mid-secretory phase, outside of which successful implantation does not occur. The endometrium also changes from a proliferative architecture to a secretory one, with maximal secretions occurring during the period of receptivity. The changes in structure and function are caused by progesterone, which regulates expression and modification of numerous protein species.3 Despite the clear relevance to endometrial function, little is known about the identity of proteins secreted by the endometrium and no specific morphological feature, nor unique gene or protein marker, has been described that effectively distinguishes between receptive and nonreceptive endometrium. Furthermore, there is no
\end{abstract}


currently available test of endometrial receptivity to guide research or clinical practice. $4^{-8}$ Thus, we used a broad-based proteomics approach to investigate changes in endometrialsecreted protein expression, which we hypothesized would provide insight into the physiology of receptivity and allow the identification of novel diagnostic and therapeutic targets.

Proteomics techniques allow the identification, characterization, and quantification of many or all proteins involved in a particular pathway, organelle, cell, tissue, organ, or organism. In comparison to messenger RNA (mRNA) expression studies, a proteomic approach has many advantages: (1) proteins, not mRNA, are the functional molecules for most relevant physiologic processes; (2) since changes in mRNA abundance are one of multiple mechanisms by which protein quantity change, variations in protein quantity do not always correlate with mRNA levels ${ }^{9}$; (3) proteins can be modified in their structure and function by posttranslational changes including proteolytic maturation, phosphorylation, and glycosylation, which can only be detected by studying the proteins directly.

A disadvantage to proteomic techniques is that they are more demanding to perform than genomic techniques as current technology requires adaptation of the investigative methods for each specimen and protein attribute. Unlike nucleic acid techniques, there are no simple methods to amplify signal analogous to polymerase chain reaction (PCR) nor large-scale survey methods analogous to DNA microarray that allow exhaustive characterization of every protein in a sample. Each of the multiple proteomic techniques available has distinct limitations and the decision of which methods to use and how to use them is largely empiric, requiring much trial and error. In this manuscript, we describe application of 2-dimensional (2D) difference gel electrophoresis (DIGE), followed by trypsin digestion and identification by mass spectrometry. The DIGE technique uses different cyanine fluorescent dyes (Cy3 \& Cy5), with nearly identical molecular mass and charge but whose emission spectra are distinct. Different samples are thus labeled with different cyanine dyes and separated on the same 2D gel to allow direct comparison of protein amount.

Initial studies using proteomic techniques to investigate endometrial tissue protein expression in normal and pathologic states have found the techniques to be feasible and useful in broadening understanding of endometrial physiology. $9-11$ However, we hypothesized that the secretome, defined as all secreted proteins, would be more informative than proteins obtained from tissue extracts because of the tight linkage between secretory histology and receptivity. Also, the reduction in numbers of protein species in lavage fluid versus tissue extracts increases the ability of proteomic techniques to separate and identify protein species. Finally, as a tool for diagnostic testing, endometrial lavage may be more clinically acceptable as a test for endometrial receptivity than biopsy, because lavage is less invasive and less traumatic than biopsy.

The objectives of this study are to begin broad characterization of the endometrial secretome and to compare differential protein expression between prereceptive and receptive-phase endometrium. Understanding the secretome and its changes during the luteal phase will promote insight into endometrial receptivity and create a foundation to further study causes of abnormal endometrial receptivity, implantation defects, and infertility.

\section{MATERIALS AND METHODS}

\section{Sample Collection and Preparation}

Endometrial lavage samples were collected from 18 to 34 year old $(\mathrm{N}=10)$, healthy, regularlycycling volunteers on no hormonal medication, under an approved Institutional Review Board protocol at the University of North Carolina. Samples were collected 4 days and 9 days after urinary luteinizing hormone $(\mathrm{LH})$ surge detection $(\mathrm{LH}+4$, collected $\mathrm{N}=10 ; \mathrm{LH}+9$, as $\mathrm{N}=13$ ). 
Lavage samples were follows: a speculum was placed in the vagina and the cervix cleansed with povidone-iodine solution. A Goldstein sonohysterography catheter (Cook Medical, Bloomington, IN) was passed through the internal cervical os and inflated with $1.5 \mathrm{~mL}$ of air. Normal saline, $4 \mathrm{~mL}$, was flushed into the uterine cavity and removed into sterile containers, twice. Samples were centrifuged for 5 minutes at $800 \mathrm{~g}$ and the supernatant was removed for analysis. Protease inhibitor $(20 \mu \mathrm{L} / \mathrm{mL}$; GE Healthcare product 80-6501-23, Piscataway, NJ) was added to the samples, which were then stored at $-80^{\circ} \mathrm{C}$ until ready for analysis.

Samples were depleted of nonprotein impurities and concentrated using the Ettan 2-D cleanup kit (GE Healthcare product 80-6484-51, Piscataway, NJ), and highly abundant serum proteins were depleted using the Agilent High Capacity Multiple Affinity Removal System (Agilent Technologies, Santa Clara, CA) or the Vivapure Anti-HSA Kit for Human Albumin Depletion (Goettingen, Germany). Sample desalting and buffer-exchange was then performed using Zeba Desalt Spin Columns (Pierce, Rockford, IL), followed by protein quantitation of each sample using the Micro BCA Protein Assay Kit (Pierce, Rockford, IL). All kits were used according to manufacturer instructions.

\section{Two-Dimensional Gel Electrophoresis for Secretome Survey}

A total of 10 lavage samples collected on day LH+4 and 10 samples collected on day LH+9 were, respectively, pooled to collectively identify the early and mid-secretory phase secretomes. From each sample, $100 \mu \mathrm{g}$ of protein were combined and placed on an Immobiline DryStrip (pH 3-10, 18-cm strip [18 samples] or pH 4-7, 18-cm strip [2-day LH+4 samples], GE Healthcare, Piscataway, NJ). There were 2 different $\mathrm{pH}$ ranges used due to the pilot nature of this study and our desire to investigate different experimental conditions and their effect on protein separation. Following equilibration of the Dry-Strip in denaturing buffers, the proteins were separated by isoelectric focusing on the Ettan IPGphor II isoelectric focuser (GE Healthcare) using the following voltage settings: $30 \mathrm{~V} \times 10$ hours, $1000 \mathrm{~V} \times 30$ minutes, $4000 \mathrm{~V}$ $\times 1$ hour, $8000 \mathrm{~V} \times 1$ hour, $8000 \mathrm{~V} \times 12$ hours, and $1000 \mathrm{~V} \times 10$ hours.

The Drystrip was then placed on a polyacrylamide gel (4\%-12\% gel [N $=13]$ or $10 \%-20 \%$ gel $[\mathrm{N}=7]$, GE Healthcare), for the second dimension of separation by molecular weight. The reason for the 2 different experimental conditions involving gel concentration was the same as the rationale for different $\mathrm{pH}$ conditions, described above. Protein spots were detected by staining with SYPRO Ruby IEF Protein Stain (Bio-Rad Laboratories, Hercules, CA) and fluorescent imaging using the Typhoon 9400 Gel Imager (Amersham Biosciences, GE Healthcare). Progenesis discovery software (Nonlinear Dynamics, Durham, NC) was used to define spot boundaries and quantitatively compare protein levels. The integrated intensity of the fluorescence over the entire spot was used as a measure of the relative amount of protein in that spot. Each protein spot identified measuring at least $1 \mathrm{~mm}^{3}$ was excised to a 96-well plate using a 2DiD gel-cutting robot (Biomachines, Research Triangle Park, NC).

The sample plate was then transferred to a ProGest Protein Digestion Station (Genomic Solutions, Ann Arbor, MI) for destaining and trypsin digestion. Samples were then lyophilized overnight and kept at $-80^{\circ} \mathrm{C}$ until ready for mass spectrometry analysis. Analysis was performed using matrix-assisted laser desorption ionization time-of-flight tandem mass spectrometry (MALDI-TOF/TOF) on an ABI 4800 instrument (Foster City, CA).

\section{Analysis of Differential Protein Expression}

LH+4 and LH+9 lavage samples were, respectively, labeled with Cy 3 and Cy 5 dye, and then pooled. Each pool was then separated by 2D gel electrophoresis as described above. The gel was then scanned at the wave-length specific for each dye (Cy 3 and $\mathrm{Cy} 5)$ using the Typhoon 9400 Gel Imager (Amersham Biosciences, GE Healthcare, Piscataway, NJ). Progenesis 
discovery software (Nonlinear Dynamics, Durham, NC) was used to overlay the images, define spot boundaries, and quantitatively compare protein levels. The integrated intensity of the fluorescence over the entire spot was used as a measure of the relative amount of protein in that spot. Each spot varying more than 2-fold was removed using the 2DiD gel-cutting robot and in-gel digested with trypsin, with peptides eluted and subjected to MALDI-TOF/TOF as described previously.

\section{Protein Identification and Statistical Analysis}

Mass spectra of peptide mixtures derived from proteolytically digested protein samples were analyzed using GPS Explorer (Applied Biosystems, Foster City, CA) and Mascot (Matrix Science, Boston, MA) software. Scores are generated based on the number of matches between observed peptide masses and masses of peptides obtained from an in-silico digest of all proteins within the given database of interest. The software also scores the quality of the tandem mass spectrometry (MSMS) peptide sequence information. Protein hits with a combined mass spectrometry (MS) mass fingerprint and MSMS ion sequence score above the significance threshold for a given database were reported as identifications. In cases where more than one protein matched the same set of peptides, only the highest scoring protein hit was reported. Protein information was obtained from the Uniprot database (http://www.pir.uniprot.org/), NCBI Entrez protein database, and the Mass Spectrometry Protein Sequence Database (http://csc-fserve.hh.med.ic.ac.uk/msdb.html).

\section{RESULTS}

Among the 23 endometrial lavage samples collected from 10 volunteers, the mean volume of fluid recovered after injection was $4.3 \mathrm{~mL}$ (range 1.0-8.0 mL). Mean protein quantitation per sample was $4.92 \mu \mathrm{g} / \mu \mathrm{L}$ (range 2.70-7.14 $\mu \mathrm{g} / \mu \mathrm{L}$ ). Seven preparative gels and eleven differential gels were run to investigate overall and differential protein expression, respectively. Analysis of the gels showed variability in the number of protein species identified, with some gels identifying as few as 2 proteins and others as many as 104 proteins. The reason for this variability may have been the varying quantities of fluid recovered from the lavage collections, biological variability, and the stringent criteria we used in the protein expect score for probability to identify significant protein matches.

Protein identities were organized by assignment to 1 or more of 3 tables: overall protein identification from the preparative gels (Table 1); proteins showing increased expression between cycle days LH+4 to LH+9 (Table 2); and proteins showing decreased expression between cycle days LH+4 to LH+9 (Table 3). Differential expression was defined as at least a 2-fold difference of integrated fluorescence on the same gel. As these are direct comparisons between 2 individuals, a significant variability in fold difference is expected between gels as each gel includes proteins from 2 unique individuals.

To simplify presentation, any proteins listed in one of the differential expression tables were not listed in the general protein identification table. Not all proteins identified in the differential analysis were identified in the overall analysis, highlighting the variability seen among DIGE gels. A total of 468 protein spots were identified among the gels and many separate spots were identified as fragments or derivatives of the same parent protein, likely indicating posttranslational processing and/or presence of multiple isoforms. These identifications were consolidated for a total of 152 unique parent proteins identified (summarized in Tables 1-3), with 64 proteins not previously reported in endometrial tissue. We assessed whether a protein had been previously reported by manually searching medline and the extant microarray studies for evidence of either mRNA or protein expression in endometrial tissue. 
Regarding functional classification, proteins related to apoptosis regulation, stress response, host defense, immune/inflammatory response, molecule transport, and ion homeostasis were most frequently represented. As a group, proteins belonging to the immunoglobulin family were most commonly identified, supporting the hypothesis that the endometrium is an important site of immune function.

The most highly upregulated proteins were involved in apoptosis regulation (glutathione transferase), coagulation ( $\alpha$-1-antitrypsin precursor, fibrinogen precursor, and kininogen 1 variant), immune response (polymeric immunoglobulin receptor precursor), molecule transport (apolipoprotein A1 fragment and transferrin), and transcription regulation (cytoplasmic polyadenylation element-BP 1). The most highly downregulated proteins were involved in stress response (heat shock protein $\beta-1$ ), cell structure (cofilin-1, desmuslin, keratin type 1 cytoskeletal 9, and keratin type 2 cytoskeletal 1), complement pathway (clusterin precursor), immune response (multiple immunoglobulins, and polymeric immunoglobulin receptor precursor), metabolism (glyceraldehyde-3-phosphate dehydrogenase), and molecule transport (apolipoprotein A1, hemoglobin $\beta$, and phosphatidylethanolamine-binding protein-1).

Some protein species were identified as having variable expression (increased in one comparison, decreased in another) between days $\mathrm{LH}+4$ and $\mathrm{LH}+9(\mathrm{~N}=13)$. These protein species included heat shock protein $\beta-1,78$ kd glucose-regulated protein precursor, $\alpha-1$ antitrypsin precursor, anti tumor necrosis factor (TNF- $\alpha$ ) antibody, haptoglobin precursor, IGKC protein, orosomucoid 1 and 2, polymeric immunoglobulin receptor precursor, apolipoprotein A1 fragment, transferrin precursor, and transferrin variant fragment. Review of the protein spots on the gels showed differing locations for some of these proteins, suggesting differing isoelectric point ( $\mathrm{pI}$ ) and molecular weight for the species identified and thus changes in posttranslational modification. Because the DIGE technique depends on spot location to determine relative change in protein expression, the altered gel migration results in the appearance of upregulation at one gel location and downregulation at the other.

\section{DISCUSSION}

This article describes the first proteomic analysis of the endometrial secretome and the first proteomic comparison of early secretory to mid-secretory endometrium. Proteins, the end products of gene expression, are functional molecules critical for virtually all physiologic processes. Although transcriptomic techniques, such as DNA microarray, allow efficient evaluation of altered expression of tens of thousands of mRNA species, changes in mRNA expression do not always correlate with alterations in protein quantity. Also, posttranslational modifications and variations in protein half-life are invisible to nucleic acid-based measurements. Thus, complete understanding of endometrial function requires examination of changes in secreted endometrial proteins.

Using 2D DIGE MS/MS analysis, more than 400 protein spots were identified in endometrial lavage fluid, including 82 differentially secreted proteins and 64 proteins not previously reported in the endometrium. The most frequently identified proteins had functions involving host defense and molecule transport, while proteins showing the greatest differential expression were involved in host defense, coagulation, apoptosis regulation, and stress response. Many proteins demonstrated apparent posttranslational modification, as the same parent protein was found in different spots, which demonstrated increase in one spot and decrease in another.

The strengths of our study include the novel techniques we used for sample collection and proteomic analysis, our larger sample size than prior proteomic investigations of endometrial tissue, and our use of a proteomics core facility with extensively trained proteomic laboratory 
technicians and investigators. The weaknesses include obtaining varying volumes of fluid from volunteers during the lavage procedure, which may affect protein concentration and detection. Additionally, we were unable to detect several proteins thought to be critical to implantation including glycodelin, leukemia inhibitory factor (LIF), and osteopontin.12-14 These proteins have molecular weights ranging from 18.7 to $28 \mathrm{kd}, 32$ to $67 \mathrm{kd}$, and 25 to $75 \mathrm{kd}$, respectively, due to extensive and variable posttranslational modifications. $15^{-} 17$ Because of the small size of some forms of these proteins, we conducted the same experiment under different conditions, changing the gel gradient of molecule separation from $4 \%-12 \%$ to $10 \%-20 \%$ in an effort to capture smaller molecules. However, we were still unable to identify these proteins. Some of these proteins are also highly acidic; however, the broad $\mathrm{pH}$ range we used for protein separation by isoelectric focusing ( $\mathrm{pH} 3-10$ ) should have captured proteins with a wide variation in pI. We suspect that either these proteins were present in small quantities in our lavage samples or the high degree of glycosylation and phosphorylation of these proteins affected our ability to detect them in the analyses. This may be a limiting feature of current proteomic techniques if altered posttranslational modification affects the ability to detect various posttranslational protein forms.

Another potential problem with our approach is possible contamination of the lavage samples with blood and/or endometrial cells. The samples were immediately placed on ice and then centrifuged within 8 minutes to remove any intact cells, but the presence of hemoglobin protein is likely due to a small amount of red blood cell contamination. On the other hand, hemoglobin mRNA has also been reported in eutopic endometrial biopsy specimens by microarray in women with early ectopic pregnancy. ${ }^{15}$ The presence of cytoskeletal proteins may also suggest endometrial cell contamination, however, another interpretation could be that endometrial epithelial cells are shed into the lumen and thus contribute intracellular proteins to the luminal fluid.

Previously, few investigators have applied large-scale proteomic techniques to study endometrial function, and each of these prior studies used endometrial biopsy specimens to extract proteins for analysis. ${ }^{12,16-18}$ Of these reports, 3 focused on endometrial cancer ${ }^{16-18}$ and none used the 2D DIGE method. A fourth study used 2D gels without DIGE labeling followed by MALDI-TOF mass spectrometry to identify proteins differentially expressed in serum and endometrial tissue in women with and without endometriosis. ${ }^{19}$ Those investigators reported identification of 11 differentially expressed serum proteins and 8 differentially expressed tissue proteins. There was no significant overlap between their findings and ours.

A report by Desouza et al is more comparable to ours, though with fewer participants, protein extracted from tissue rather than lavage, different proteomic techniques, and comparison between proliferative and secretory phase endometrium. ${ }^{10}$ Nonetheless, Desouza et al identified 28 proteins identical to those identified by our methods, providing some validation to both approaches. The common proteins identified by both studies were actin, alcohol dehydrogenase, $\alpha$-2-HS-glycoprotein, $\beta$-2-glycoprotein I, cofilin, complement $\mathrm{C} 3$ precursor, creatine kinase B, enolase, fibrinogen $\gamma$ chain, gelsolin, glutathione S-transferase, glyceraldehyde 3-phosphate dehydrogenase, haptoglobin, heat shock cognate $71 \mathrm{kd}$ protein, hemoglobin $\beta$, hemopexin precursor, immunoglobulin (Ig) $\gamma 2$ chain, Igא chain, phosphatidylethanolamine-binding protein, polymeric Ig receptor precursor, transferrin, albumin, superoxide dismutase, triosephosphate isomerase, tropomyosin $\alpha 4$ chain, tubulin $\beta$ chain, and vitamin D-binding protein precursor. ${ }^{10}$

Whereas the overlap in proteins identified provides some substantiation of the use of both techniques, differences are also expected as our study examined secreted proteins rather than proteins extracted from tissue composed of many cell types. By examining endometrial tissue specimens using the iCAT technique (which uses differential isotopic labeling to quantitate 
protein species), Desouza et al were able to identify 119 proteins differentially expressed between proliferative and secretory endometrium, which is more than the 83 differentially expressed proteins between early and mid-secretory phases identified by the current study. ${ }^{10}$ Possible explanations for differences in yield include differences in cycle phases compared, the presence of more protein species in a biopsy specimen than in lavage fluid, and differences between separation and analysis techniques.

A recent report by Parmar et al using lower-throughput, manual, 2D gel techniques to compare protein from proliferative to mid-secretory endometrial tissue identified an increase in $\alpha-1$ antitrypsin similar to the increase we detected between the early and mid-secretory phase in our study. ${ }^{20}$ They also identified heat shock protein $\beta-1$ (heat-shock protein 27 ) and transferrin as upregulated in endometrial tissue and present in uterine fluid and mid-secretory secretions. We found that these 2 proteins appeared upregulated in one form and downregulated in another, suggesting changes in posttranslational modification.

A particularly interesting finding in both Desouza's articles and ours is the upregulation of fibrinogen $\gamma$ chain protein.10,20 Desouza et al found only a 1.2-fold increase in fibrinogen $\gamma$ chain protein quantity between the proliferative and secretory phase, while we found a 4- to 5 -fold increase between the early and mid-secretory phase. In contrast, Parmar et al showed a downregulation of fibrinogen $\beta$ chain from the proliferative to mid-secretory phase. ${ }^{20}$

Unpublished data in baboons suggest the importance of differential regulation of endometrial fibrinogen expression in endometriosis. Mid-secretory phase endometrial lavage fluid collected from baboons with endometriosis shows markedly upregulated fibrinogen $\beta$ secretion compared to lavage fluid collected from baboons without endometriosis (Fazleabas A, Personal communication December 2008). In light of the interesting findings by others in regard to fibrinogen $\beta$, we have checked all of the spectra identifying fibrinogen $\gamma$ and in no instance could that peptide be alternatively identified as fibrinogen $\beta$. Taken together, these data suggest the hypothesis that alterations in secretion of fibrinogen subunits may reflect important physiological changes in endometrium.

The current study may also be compared with previous genomic studies of endometrial RNA expression. The most comprehensive genomic study available used DNA microarray to determine that 1415 genes were upregulated and 1463 genes were down regulated between the early to mid-secretory phase. ${ }^{21}$ Others have found many genes changing over the same cycle phases, though with some differences between studies.21-23 Our findings of significant increases in proteins involved in host defense and immune response and molecule transport from the early to mid-secretory phase corroborates the increased gene expression seen among genes involved with these biological processes by prior investigators. $21^{-23}$ Although there were some similarities in categories of biological processes we found to have decreased protein expression compared to prior microarray data (cell motility, metabolism and ion homeostasis), we also detected many downregulated proteins important in host defense, immunity, and stress response, categories that were not previously shown to be downregulated.

We found several specific proteins correlating with gene identifications among prior microarray studies including Ig $\lambda$, tropomyosin, tubulin, Ras association domain protein, ceruloplasmin, and clusterin. ${ }^{21-} 23$ Although our findings of increased Ras association domain protein from the early to mid-secretory phase agreed with previously described increased gene expression, 23 our findings of decreased clusterin and tubulin protein expression differed from prior microarray analyses which found increased RNA expression of these genes.21, $22 \mathrm{We}$ were unable to detect many of the expected protein products from the most highly upregulated genes detected in prior studies (apolipoprotein E, phospholipase A2, glucuronyltransferase I, glycodelin, osteopontin, and decay accelerating factor). ${ }^{22,23} \mathrm{~A}$ possible explanation of this discrepancy is that many of the most highly upregulated genes in the microarray studies 
represent low abundance and/or low-molecular-weight proteins, for which the DIGE technique has low sensitivity.

In summary, proteomic analysis of endometrial lavage samples is a novel and useful technique to define the endometrial secretome and explore differential protein secretion. The identification of more than 400 protein species and the detection of differentially secreted and novel proteins suggest that proteomic techniques are powerful tools to broaden our understanding of endometrial physiology. Work is ongoing to determine the use of this technique for identifying markers of endometrial receptivity and endometrial disease.

\section{Acknowledgments}

We gratefully acknowledge the help of the National SCCPIR Center for Proteomics Director, Dr. Carol E. Parker; Assistant Director, Dr. Maria Warren Hines; as well as the Center's staff: Dr. David W. Robinette, Ms. Nedyalka N. Dicheva, and the late Dr. Viorel Mocanu, and for their expert assistance and advice. We also acknowledge the critical assistance of Amy Brown in recruitment, coordination, and cataloguing of human participants and collection of specimens. The study was supported by the Eunice Kennedy Shriver NICHD/NIH through cooperative agreement U54 HD035041 as part of the Specialized Cooperative Centers Program in Reproduction and Infertility Research. Additional financial support was provided by the UNC Nova Carta Foundation.

The study was conducted at the University of North Carolina at Chapel Hill.

\section{REFERENCES}

1. Webb, PD.; Glasser, SR. Implantation. In: Wolf, DP.; Quigley, MM., editors. Human In Vitro Fertilization and Embryo Transfer. Plenum Press; New York, NY: 1984. p. 341

2. Levi Setti PE, Colombo GV, Savasi V, et al. Implantation failure in assisted reproductive technology and a critical approach to treatment. Ann N Y Acad Sci 2004;1034:184-199. [PubMed: 15731311]

3. Norwitz ER, Schust DJ, Fisher SJ. Implantation and survival of the early pregnancy. N Engl J Med 2001;345(19):1400-1408. [PubMed: 11794174]

4. Noyes RW, Hertig AW, Rock J. Dating the endometrial biopsy. Fertil Steril 1950;1:3-25.

5. Duggan MA, Brashert P, Ostor A, et al. The accuracy and interobserver reproducibility of endometrial dating. Pathology 2001;33(3):292-297. [PubMed: 11523927]

6. Murray MJ, Meyer WR, Zaino RJ, et al. A critical analysis of the accuracy, reproducibility, and clinical utility of histologic endometrial dating in fertile women. Fertil Steril 2004;81(5):1333-1343.

[PubMed: 15136099]

7. Coutifaris C, Myers ER, Guzick DS, et al. Histological dating of timed endometrial biopsy tissue is not related to fertility status. Fertil Steril 2004;82(5):1264-1272. [PubMed: 15533340]

8. Myers ER, Silva S, Barnhart K, et al. Interobserver and intraobserver variability in the histological dating of the endometrium in fertile and infertile women. Fertil Steril 2004;82(5):1278-1282. [PubMed: 15533342]

9. Kyama CM, T'Jampens D, Mihalyi A, et al. ProteinChip technology is a useful method in the pathogenesis and diagnosis of endometriosis: a preliminary study. Fertil Steril 2006;86(1):203-209. [PubMed: 16716317]

10. DeSouza L, Diehl G, Yang EC, et al. Proteomic analysis of the proliferative and secretory phases of the human endometrium: Protein identification and differential protein expression. Proteomics 2005;5(1):270-281. [PubMed: 15602768]

11. Zhang H, Niu Y, Feng J, Guo H, Ye X, Cui H. Use of proteomic analysis of endometriosis to identify different protein expression in patients with endometriosis versus normal controls. Fertil Steril 2006;86(2):274-282. [PubMed: 16750201]

12. Seppälä M, Koistinen H, Koistinen R, et al. Glycodelins as regulators of early events of reproduction. Clin Endocrinol (Oxf) 1997;46(4):381-386. [PubMed: 9196597]

13. Cullinan EB, Abbondanzo SJ, Anderson PS, Pollard JW, Lessey BA, Stewart CL. Leukemia inhibitory factor (LIF) and LIF receptor expression in human endometrium suggests a potential autocrine/ 
paracrine function in regulating embryo implantation. Proc Natl Acad Sci U S A 1996;93(7):31153220. [PubMed: 8610178]

14. Johnson GA, Burghardt RC, Bazer FW, Spencer TE. Osteopontin: roles in implantation and placentation. Biol Reprod 2003;69(5):1458-1471. [PubMed: 12890718]

15. Savaris RF, Hamilton AE, Lessey BA, Guidice LC. Endometrial gene expression in early pregnancy: lessons from human ectopic pregnancy. Reprod Sci 2008;15(8):797-816. [PubMed: 18591649]

16. Yang EC, Guo J, Diehl G, et al. Protein expression profiling of endometrial malignancies reveals a new tumor marker: chaperonin 10. J Proteome Res 2004;3(3):636-643. [PubMed: 15253447]

17. Yoshizaki T, Enomoto T, Nakashima R, et al. Altered protein expression in endometrial carcinogenesis. Cancer Lett 2005;226(2):101-106. [PubMed: 16039949]

18. DeSouza LV, Grigull J, Ghanny S, et al. Endometrial carcinoma biomarker discovery and verification using differentially tagged clinical samples with multidimensional liquid chromatography and tandem mass spectrometry. Mol Cell Proteomics 2007;6(7):1170-1182. [PubMed: 17374602]

19. Zhang H, Niu Y, Feng J, Guo H, Ye X, Cui H. Use of proteomic analysis of endometriosis to identify different protein expression in patients with endometriosis versus normal controls. Fertil Steril 2006;86(2):274-282. [PubMed: 16750201]

20. Parmar T, Gadkar-Sable S, Savardekar L. Protein profiling of human endometrial tissues in the midsecretory and proliferative phases of the menstrual cycle. Fertil Steril. 2008 Epub ahead of print.

21. Talbi S, Hamilton AE, Vo KC, et al. Molecular phenotyping of human endometrium distinguishes menstrual cycle phases and underlying biological processes in normo-ovulatory women. Endocrinology 2006;147(3):1097-1121. [PubMed: 16306079]

22. Kao LC, Tulac S, Lobo S, et al. Global gene profiling in human endometrium during the window of implantation. Endocrinology 2002;143(6):2119-2138. [PubMed: 12021176]

23. Mirkin S, Arslan M, Churikov D, et al. In search of candidate genes critically expressed in the human endometrium during the window of implantation. Hum Reprod 2005;20(8):2104-2117. [PubMed: 15878921] 


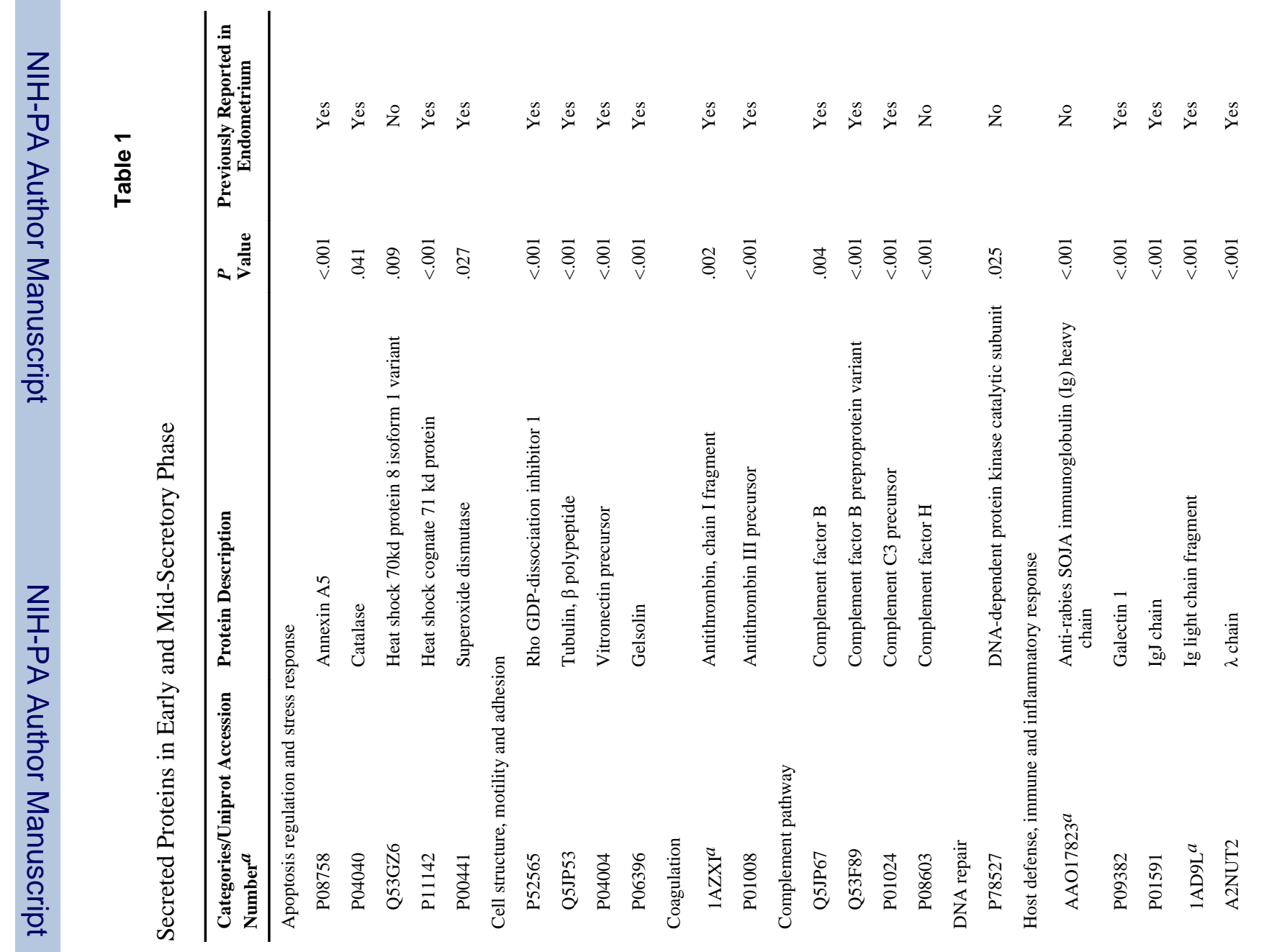

Reprod Sci. Author manuscript; available in PMC 2010 September 1. 


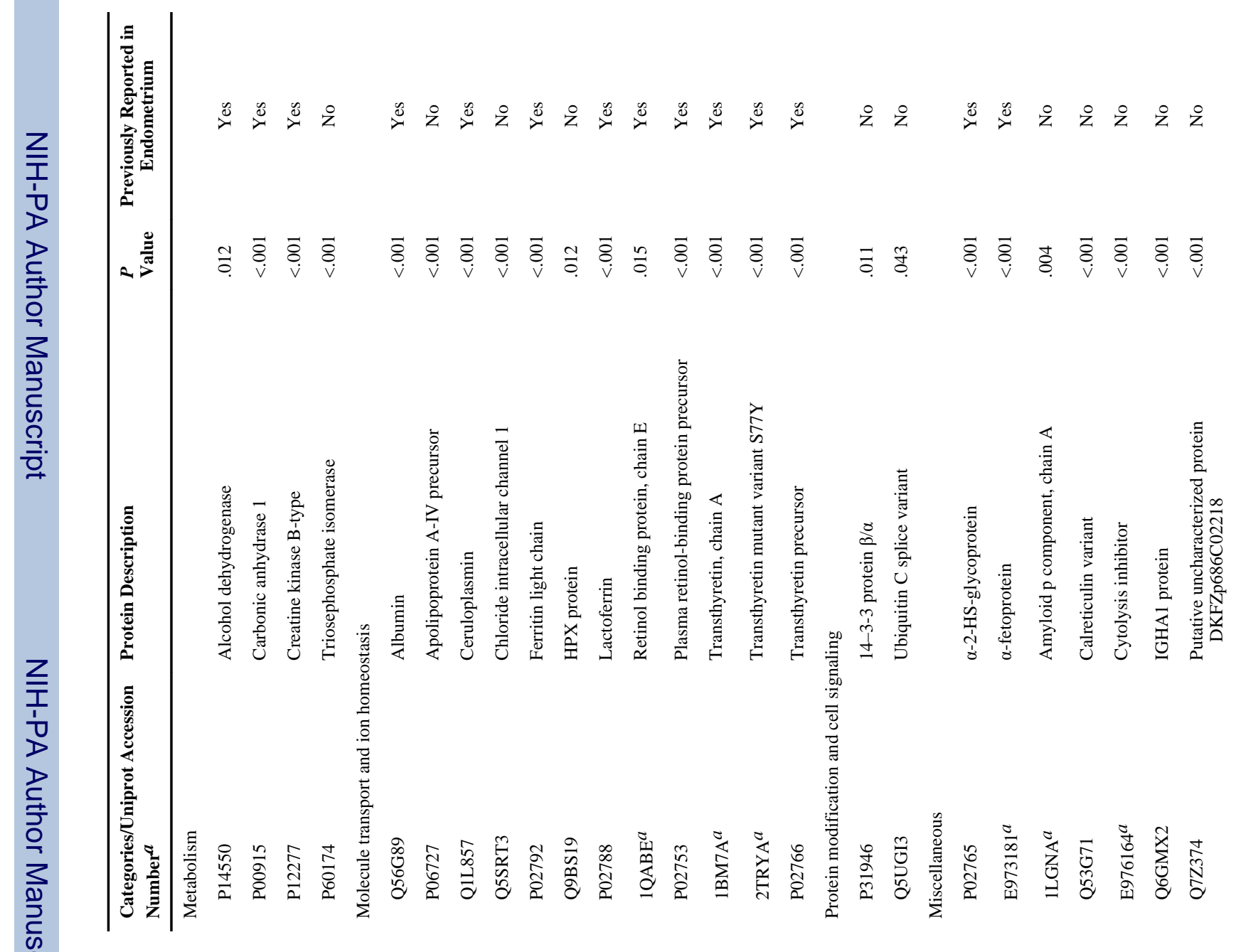




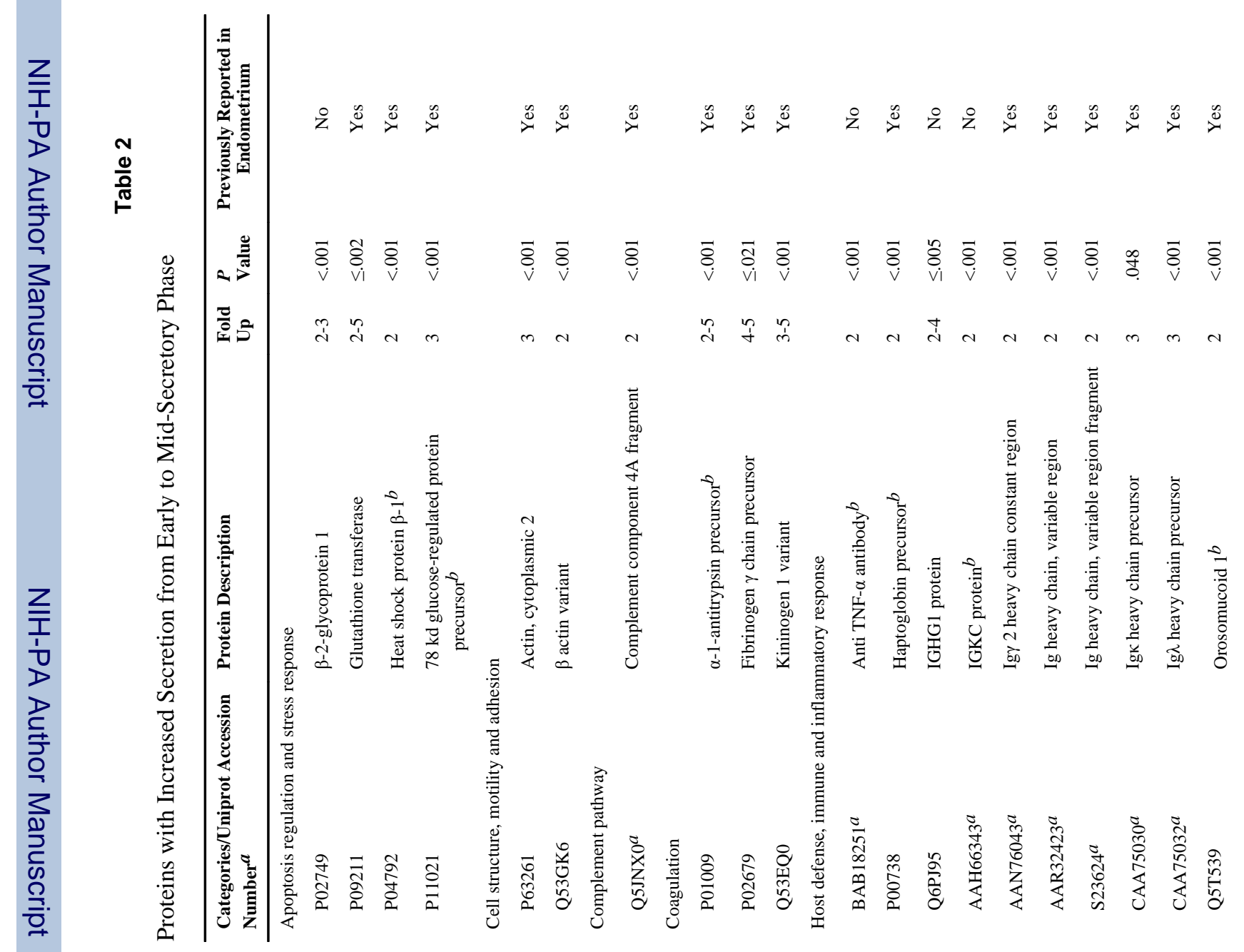

Reprod Sci. Author manuscript; available in PMC 2010 September 1. 


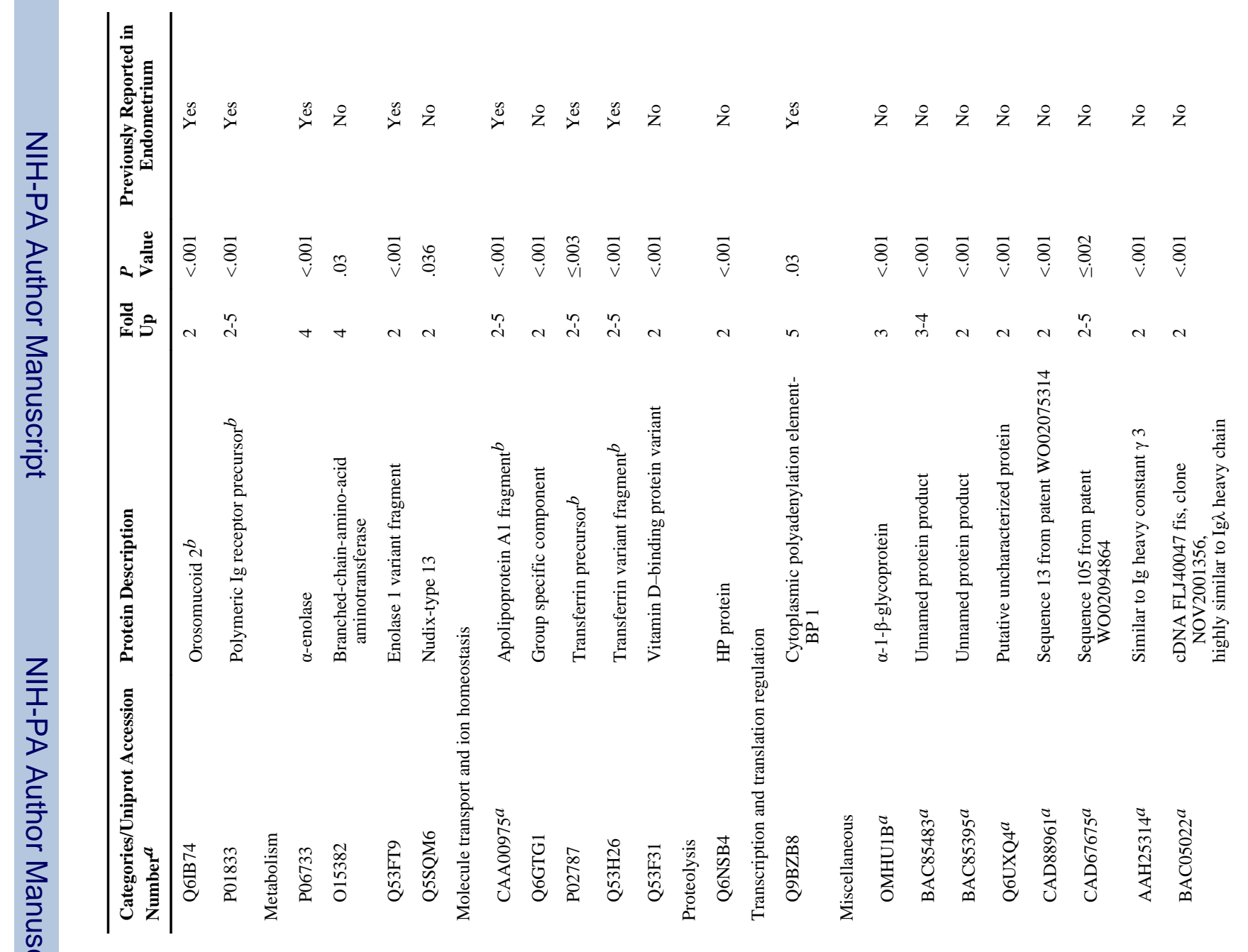




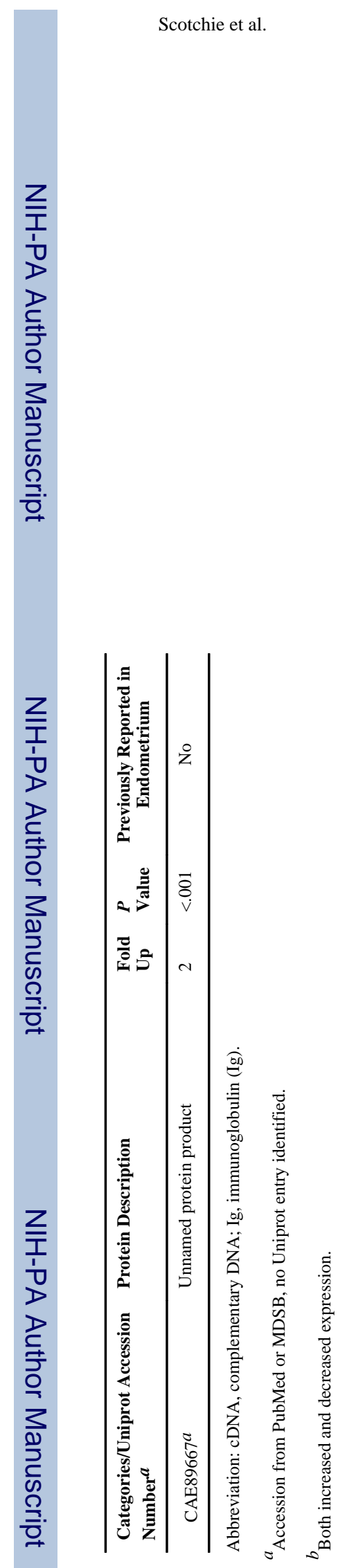

Reprod Sci. Author manuscript; available in PMC 2010 September 1. 


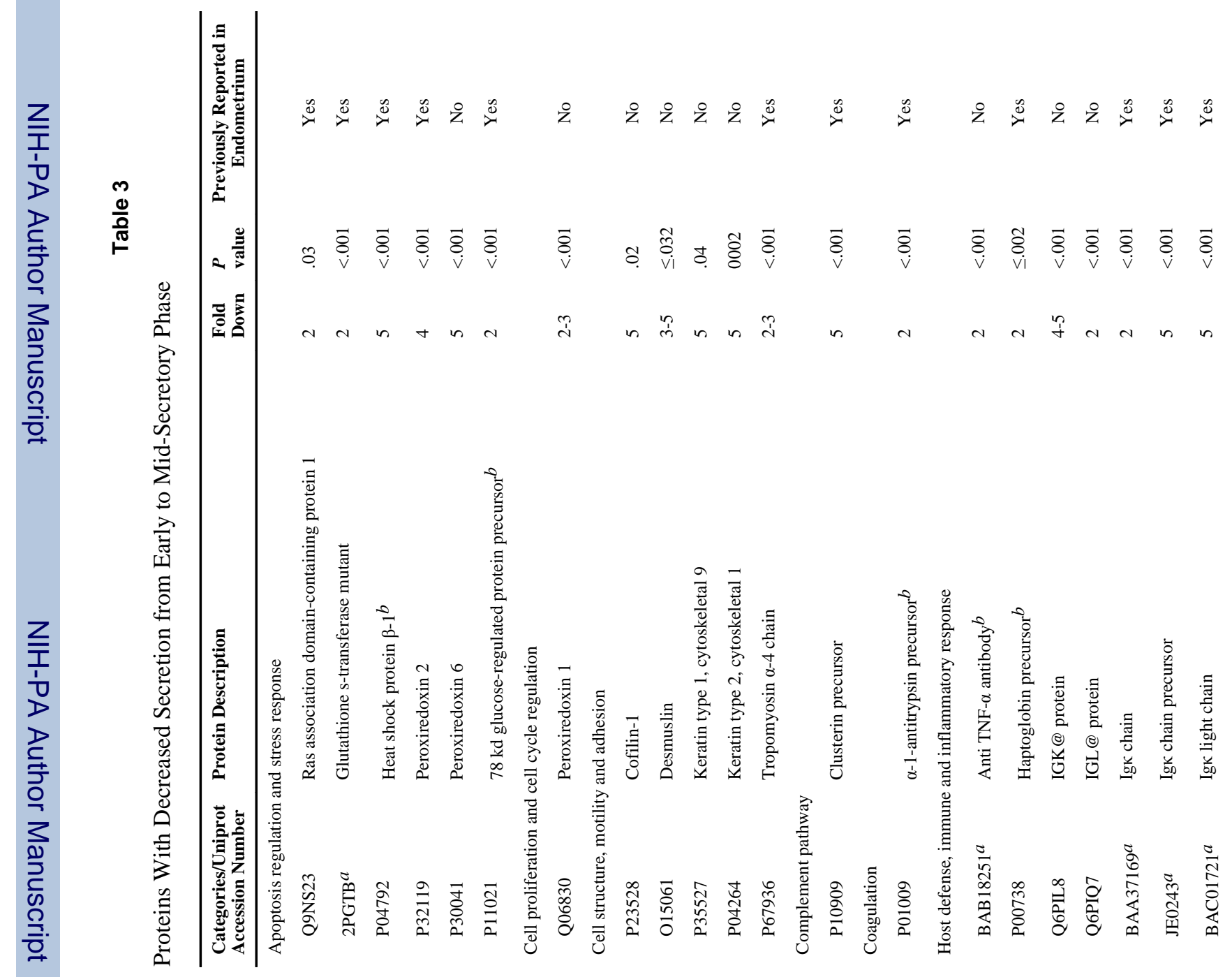

Reprod Sci. Author manuscript; available in PMC 2010 September 1. 


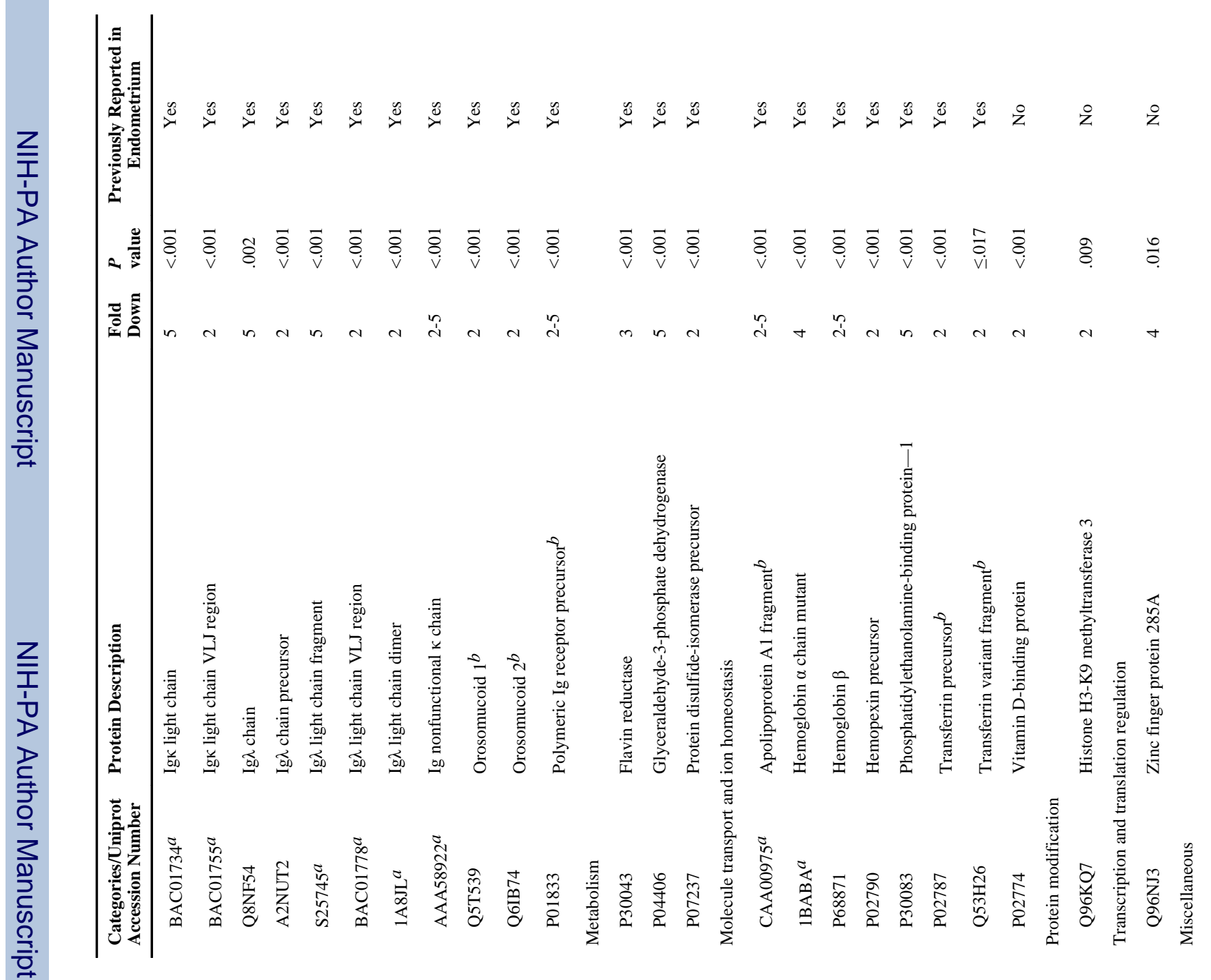


